\title{
MG53: Biological Function and Potential as a Therapeutic Target
}

\author{
Yan Zhang, Hong-Kun Wu, Fengxiang Lv, and Rui-Ping Xiao \\ State Key Laboratory of Membrane Biology, Institute of Molecular Medicine (Y.Z., H.-K.W., F.L., R.-P.X.), Peking-Tsinghua \\ Center for Life Sciences (R.-P.X.), and Beijing City Key Laboratory of Cardiometabolic Molecular Medicine (R.-P.X.), Peking \\ University, Beijing, China
}

Received January 4, 2017; accepted April 12, 2017

\begin{abstract}
MG53 (also known as tripartite motif, TRIM72) is a cardiac and skeletal muscle-specific TRIM-family protein that exhibits multiple biologic functions. First, MG53 participates in plasma membrane repair of the heart, skeletal muscle, and, other tissues. Second, MG53 is essentially involved in the cardioprotection of cardiac ischemic, preconditioning, and postconditioning by activating the PI3K-Akt-GSK3 $\beta$ and ERK1/2 survival signaling pathways. Moreover, systemic delivery of recombinant MG53 protein ameliorates the impact of a range of injury insults on the heart, skeletal muscle, lung, kidney, skin, and brain. It is noteworthy that chronic
\end{abstract}

upregulation of MG53 induces insulin resistance and metabolic diseases, such as type 2 diabetes and its cardiovascular complications, by acting as an E3 ligase to mediate the degradation of insulin receptor and insulin receptor substrate-1. In addition, MG53 negatively regulates myogenesis. In summary, MG53 is a multifunctional protein involved in the vital physiologic and pathologic processes of multiple organs and is a promising therapeutic target for various human diseases. In this review, we comprehensively summarize current research progress on the biologic functions and therapeutic potential of MG53.

\section{Introduction}

Mitsugumin 53 (MG53) was cloned in 2005 using an immunoproteomic library with the aim of identifying proteins involved in myogenesis, calcium signaling, and striated muscle integrity (Weisleder et al., 2008). MG53 is highly conserved among different species (Fig. 1) and is expressed predominantly in cardiac and skeletal muscle. Since the first report on its biologic function in 2009 (Cai et al., 2009a), MG53 has been implicated in the regulation of multiple physiologic and pathologic functions of various tissues through different mechanisms.

In 2009, MG53 was originally reported to be essential for the maintenance of skeletal muscle plasma membrane integrity (Cai et al., 2009a). The cardiac function of MG53 was first investigated by Cao and colleagues (2010), who found that, in addition to membrane repair, MG53 is a key mediator of intracellular cardioprotective signaling pathway and

This work was supported by the National Basic Research Program of China [Grants 2013CB531200 and 2012CB518000], the National Natural Science Foundation of China [Grants 31671177, 81170100, 81370234, 81630008, 81270190, and 31521062], and National Science and Technology Major Projects for Major New Drugs Innovation and Development [Grant 2013ZX09508104].

https://doi.org/10.1124/mol.117.108241. indispensable for cardiac ischemic preconditioning protection (Cao et al., 2010); however, recent studies from our laboratory, and others have demonstrated that MG53 acts as an E3 ligase to degrade the insulin receptor (IR) and insulin receptor substrate-1 (IRS-1) and subsequently induces insulin resistance and metabolic syndrome (MetS) (Song et al., 2013; Yi et al., 2013), along with their cardiovascular complications (Liu et al., 2015a). Thus, MG53 has both beneficial and detrimental functions, and its dysregulation is involved in the pathogenesis of various human diseases. In this review, we intend to provide a systemic summary of the recent progresses of MG53 research, emphasizing its biologic function and potential therapeutic applications.

\section{Structure and Expression of MG53}

MG53 has a typical tripartite motif in its $\mathrm{N}$ terminus and belongs to the TRIM (tripartite motif) family. To date, more than 70 known TRIM family members have been identified in mammalian species. These proteins play important roles in a wide range of biologic processes, including cell proliferation, differentiation, apoptosis, development, and innate immunity (Ozato et al., 2008; Doyle et al., 2010; Hatakeyama, 2011). Dysregulation of TRIM proteins is associated with diverse

ABBREVIATIONS: CaV3, caveolin-3; FAK, focal adhesion kinase; HFD, high-fat diet; IGF-1, insulin-like growth factor-1; IPC, ischemic preconditioning; I/R, ischemia/reperfusion; IR, insulin receptor; IRS-1, insulin receptor substrate-1; MEF2, myocyte enhancer factor-2; MetS, metabolic syndrome; MG53, mitsugumin 53; RISK, reperfusion injury salvage kinase; RY, ryanodine; SAFE, survivor activation factor enhancement; SPRY domain, a sequence repeat in the dual-specificity kinase SpIA and $\mathrm{Ca}^{2+}$-release channel ryanodine receptors; TRIM, tripartite motif. 


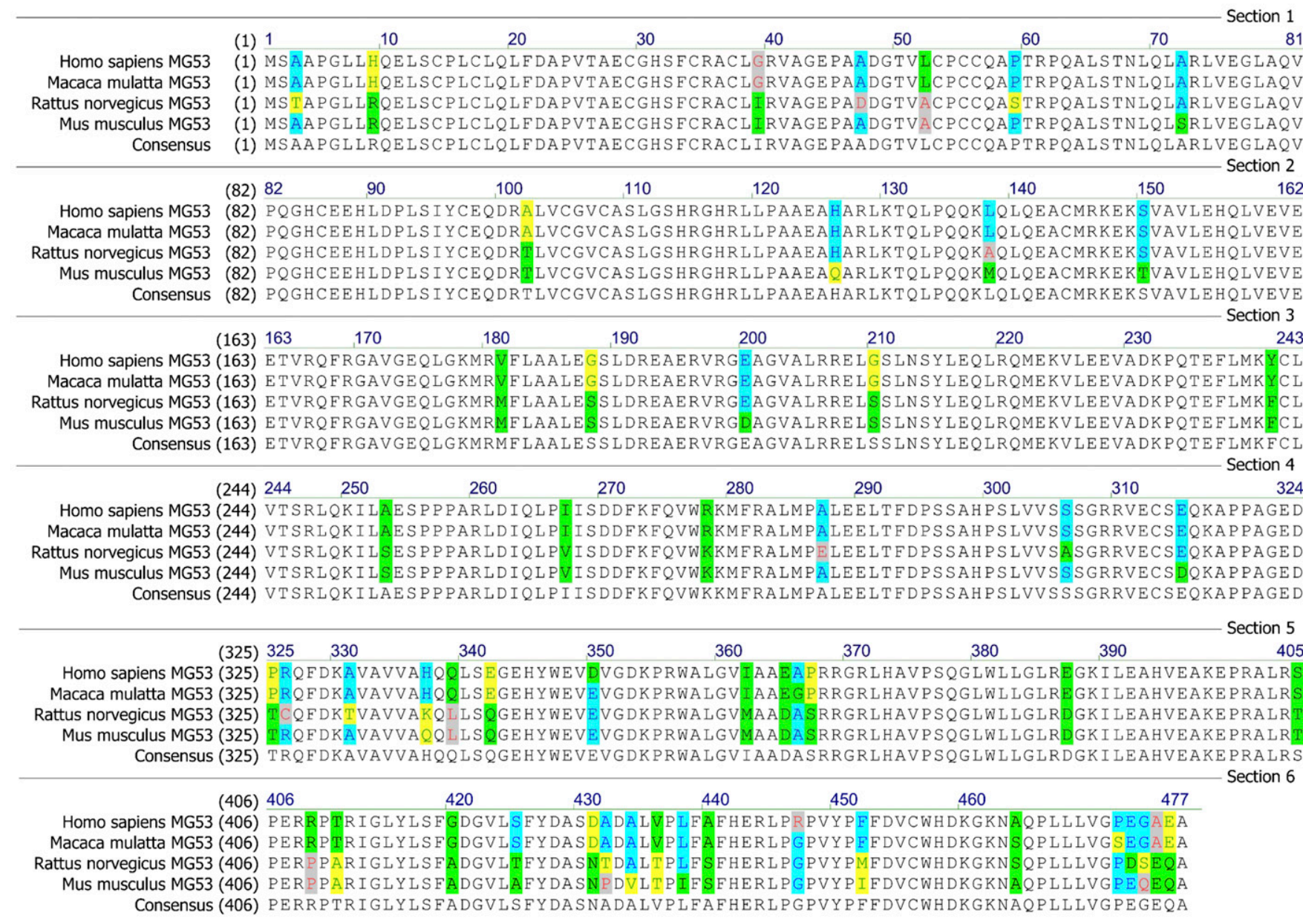

Fig. 1. Alignment of the amino acid sequence of MG53. The amino acid sequences of the MG53 protein from human (NP_001008275.2), monkey (XP_014981480.1), rat (NP_001071143.1), and mouse (NP_001073401.1) were obtained from the National Center for Biotechnology Information protein database (http://www.ncbi.nlm.nih.gov/protein). The conserved sites are highlighted with color, and the consensus sequence is shown at the bottom.

pathologic situations, including developmental disorders, viral infection, neurodegenerative diseases, and cancer (Meroni and Diez-Roux, 2005; Ozato et al., 2008; Munir, 2010; Hatakeyama, 2011; Li et al., 2014).

Similar to other TRIM members, MG53 is composed of a prototypical tripartite motif (including RING, B-box, and coiled-coil regions, also known as an RBCC domain) at its $\mathrm{N}$ terminus and a SPRY domain [a sequence repeat in the dualspecificity kinase SplA and $\mathrm{Ca}^{2+}$-release channel ryanodine (RY) receptors] at its $\mathrm{C}$ terminus (Cai et al., 2009a). The structure of MG53 is shown in Fig. 2. The RING finger domain, which is a characteristic signature of E3 ubiquitin ligases and mediates the ubiquitination events (Lorick et al., 1999), is a zinc-finger motif that contains a $\mathrm{Cys}_{3} \mathrm{HisCys}_{4}$ amino acid motif and binds with two zinc cations. The B-box domain is another zinc-binding motif, and its specific function remains unclear. The coiled-coil domain mediates monomeric and heteromeric interactions among TRIM proteins, especially for their self-associations. The SPRY domain is responsible for binding to specific target protein(s), conveying the selectivity and specificity of its E3 activity. The biologic functions of the different domain of MG53, together with its potential post-translational modifications, are summarized in Fig. 2.
The amino acid sequence of MG53 is highly conserved among different species, including human, monkey, rat, and mouse (Fig. 1). MG53 was originally regarded as a musclespecific protein. It is highly expressed in the heart and skeletal muscle and participates in multiple physiologic and pathologic processes (Cai et al., 2009a; Cao et al., 2010; Wang et al., 2010; Zhang et al., 2011; Song et al., 2013; Yi et al., 2013). Later studies have shown that MG53 is also present in the kidney and lung, although its abundance in these tissues is much lower than that in heart and skeletal muscle (Jia et al., 2014; Kim et al., 2014; Duann et al., 2015). Even in tissues with little (kidney and lung) or no (skin and brain) MG53 expression, endogenous and exogenous MG53 may have biologic functions, in particular for membrane repair (Weisleder et al., 2012; Jia et al., 2014; Kim et al., 2014; Duann et al., 2015; Li et al., 2015; Liu et al., 2015b; Yao et al., 2016). These recent findings further enhance the potential role of MG53 as a therapeutic target in human diseases.

\section{MG53 in Skeletal Muscle}

MG53 Promotes Membrane Repair of Skeletal Muscle. MG53 was first reported to be a central component of the plasma membrane repair machinery (Cai et al., 2009a). Since 
Oxidation of this site of MG53 prevents its degradation (Kohr et al., 2014 ).

Essential for the E3 ligase activity of MG53 (Song et al., 2013; Yi et al., 2013).

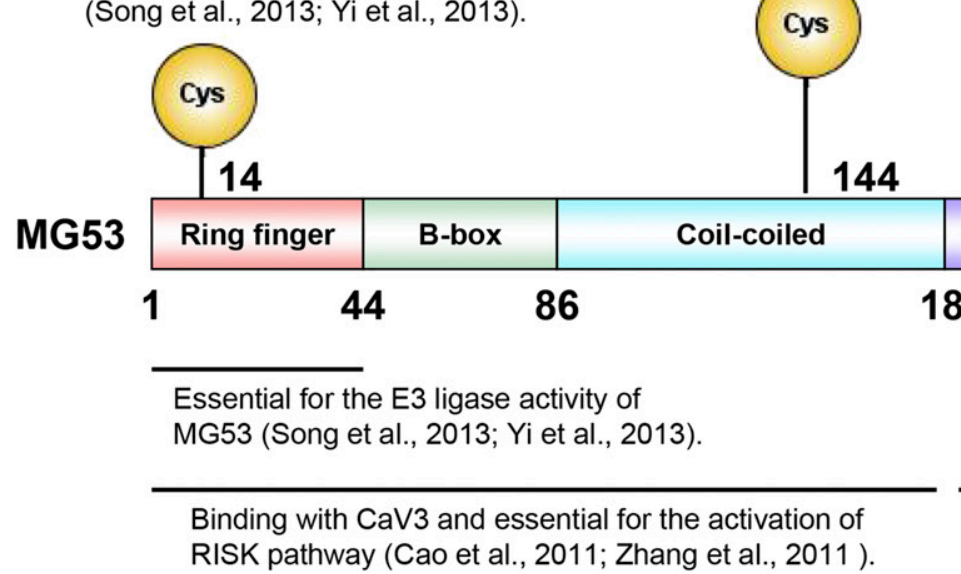

Essential for the membrane repair function of MG53 (Cai et al., 2009a; Hwang et al., 2011)
Binding with p85-PI3K and essential for the activation of RISK pathway (Cao et al., 2011; Zhang et al., 2011).

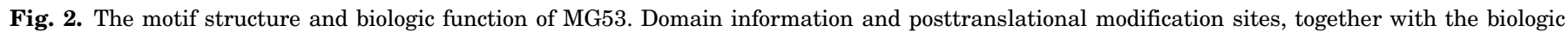
functions of mouse MG53, are depicted based on current reports.

striated muscle cells undergo severe membrane stress in response to muscle contraction, acute muscle membrane repair is particularly important. Deficiency of membrane repair causes muscle cell death, muscular injury, and dystrophy (Bansal et al., 2003; Bansal and Campbell, 2004; Han and Campbell, 2007). Upon muscular membrane rupture, MG53 senses the oxidized milieu from the extracellular space, interacts with phosphatidylserine, and localizes to the site of muscle fiber disruption, where it promotes plasma membrane resealing (Cai et al., 2009a). The MG53-mediated membrane repair process is regulated by multiple factors. $\mathrm{Ca}^{2+}$ is required for the fusion of vesicles with the plasma membrane, but not for vesicle trafficking (Cai et al., 2009a). The binding of MG53 to $\mathrm{Zn}^{2+}$ is indispensable for the membrane repair machinery (Cai et al., 2015). Mutation at cysteine 242 residue in MG53, which prevents oxidation-mediated oligomerization, impairs its membrane repair ability (Cai et al., 2009a; Hwang et al., 2011). The interaction of MG53 with dysferlin and caveolin-3 (CaV3) is essential for the cell membrane repair (Cai et al., 2009b).

The therapeutic potential of MG53-dependent prevention of skeletal muscle injury has been validated in multiple preclinical animal models. Systemic delivery of an adenoassociated virus overexpressing human MG53 protects skeletal muscle damage in a genetic muscular dystrophy hamster model through enhancement of membrane repair, activation of cell survival kinases, and inhibition of cell apoptosis (He et al., 2012). Furthermore, intravenous injection of MG53 recombinant protein ameliorates skeletal muscle damage in response to multiple insults, including toxic compounds (cardiotoxin VII4), genetic muscle diseases ( $m d x$ mice), and ischemia/reperfusion (I/R) and burn injuries (Weisleder et al., 2012; Zhu et al., 2015; Wang et al., 2016).

In contrast, it was reported that recombinant MG53 failed to protect against I/R-induced skeletal muscle injury in rats (Corona et al., 2014). The authors attributed this discrepancy (compared with data from other species) to either the more severe injury in this model, which was beyond therapeutic benefit, or to species differences (Corona et al., 2014). The serum concentrations of MG53 in mouse and human are significantly lower than that in rat, which makes rat muscle less sensitive to the therapeutic effect of recombinant MG53 (Zhu et al., 2015).

Based on the preceding data, although more work is required to fully elucidate its effects and mechanisms, MG53 is a molecular and important target with respect to therapeutic benefit in human skeletal muscle injury. As outlined in the following section, however, in other contexts, MG53 may confer detrimental effects.

MG53 Contributes to Skeletal Muscle Insulin Resistance. MetS, which is a cluster of disorders including central obesity, dyslipidemia, and hypertension, increases the risk of cardiovascular diseases and type 2 diabetes and has become one of the most serious threats to human health (Grundy, 2006). Insulin resistance has been proven to be central to the mechanism of MetS (Eckel et al., 2005; McMillen and Robinson, 2005). Previous studies on the development of global insulin resistance have focused mainly on liver and adipose tissue; however, the role of skeletal muscle, which accounts for 70\%-90\% of insulin-stimulated glucose disposal (DeFronzo et al., 1981; Shulman et al., 1990), has been largely neglected.

Our recent studies have demonstrated that skeletal muscle insulin resistance is the initial and central pathologic process during the development of global metabolic disorders. As early as 1 week after high-fat diet (HFD) treatment, skeletal muscle, but not liver and fat, exhibits profound insulin resistance (Song et al., 2013). More importantly, deletion of MG53, which is highly expressed in striated muscle, can prevent systemic insulin resistance and metabolic disorders that are induced by overnutrition, including obesity, hypertension, hyperglycemia, hyperinsulinemia, dyslipidemia, and hepatosteatosis (Song et al., 2013). Furthermore, overexpression of MG53 in striated muscle is sufficient to elicit global insulin resistance and other metabolic disorders (Song et al., 2013), indicating the central role of MG53 in systemic insulin resistance and MetS. The important role of MG53 in skeletal muscle insulin resistance has been independently confirmed by another group (Yi et al., 2013). Also, a recent report has yielded preliminary evidence that MG53 may be involved in 
the protective effect of exercise against HFD-induced insulin resistance (Qi et al., 2016).

Post-transcriptional downregulation of IR and IRS-1 is a common feature of insulin resistance in both animal models and humans (Olefsky et al., 1976; Goodyear et al., 1995; Kerouz et al., 1997; Song et al., 2013); however, the mechanisms underlying this process remained unclear until recent reports that MG53 acts as an E3 ligase to mediate the degradation of IR and IRS-1 (Song et al., 2013). Ubiquitination is a versatile post-translational modification in eukaryotic cells that controls protein abundance through proteasomemediated proteolysis (Huibregtse et al., 1995; Scheffner et al., 1995; Hershko and Ciechanover, 1998). Ubiquitin conjugation is catalyzed by ubiquitin-activating enzyme (E1), ubiquitinconjugating enzyme (E2), and ubiquitin ligase (E3); E3 ligase is responsible for the recognition of specific substrates. The RING finger domain of MG53 at its N terminal is a characteristic feature of a family of E3 ligases (Joazeiro and Weissman, 2000). Song and colleagues showed that MG53 is both sufficient and necessary for HFD-induced ubiquitination and degradation of IR and IRS-1 in skeletal muscle (Song et al., 2013). The RING domain and the cysteine 14 residue in this domain are indispensable for the E3 ligase activity of MG53 (Fig. 2) (Song et al., 2013). The E2-conjugating enzyme that helps MG53 to degrade IRS-1 was identified as UBE2H by another group, although this study failed to confirm ubiquitination and degradation of IR by MG53 (Yi et al., 2013).

Previous studies have shown that muscle-specific knockout of neither IRS-1 nor insulin receptor substrate-2 in mice causes any obvious dysregulation of glucose homeostasis (Long et al., 2011). Instead, mice with an approximately 50\% reduction of both IR and IRS-1, but not either alone, develop profound MetS (Kido et al., 2000). If MG53 selectively downregulates IRS-1, but not IR, in skeletal muscle, it is not expected that transgenic overexpression of MG53 would lead to systemic insulin resistance and metabolic disorders (Song et al., 2013). Instead, the phenotype of MG53 transgenic mice is quite similar to those with a 50\% reduction of both IR and IRS-1 (Kido et al., 2000; Song et al., 2013). Therefore, we conclude that MG53 mediates the degradation of both IR and IRS-1, resulting in systemic insulin resistance and subsequently whole-body MetS (Song et al., 2013), similar to the phenotype of the mice with an approximately $50 \%$ reduction of both IR and IRS-1.

In summary, MG53 is a novel E3 ligase, highly expressed in skeletal muscle, that targets both IR and IRS- 1 for ubiquitindependent degradation. Overexpression of MG53 causes systemic insulin resistance and metabolic disorders, constituting a central mechanism responsible for the regulation of insulin sensitivity. Recently, a small molecule that disrupts the interaction between MG53 and IRS-1 and subsequent IRS-1 ubiquitination has been shown to improve insulin sensitivity in cultured myotubes (Lee et al., 2016), highlighting that targeting MG53 E3 ligase activity is a novel strategy to sensitize insulin response and treat MetS.

MG53 Inhibits Myogenesis. Normal myogenesis is essential for the maintenance of skeletal muscle mass and function. When mature muscle is damaged, myogenesis takes place to repair the tissues through satellite cell activation and differentiation into new fibers (Chargé and Rudnicki, 2004; Rudnicki et al., 2008). Insulin-like growth factor-1 (IGF-1) is a key regulator of the differentiation of satellite cells and of the development, hypertrophy, and regeneration of skeletal muscle (Baker et al., 1993; Powell-Braxton et al., 1993; Glass, 2003). It is noteworthy that MG53 ablation preferentially targets IRS-1 with respect to the role of insulin signaling relative to IGF-1 signaling (Song et al., 2013), although IRS-1 is the shared mediator of both the IR and IGF-1 signaling pathways.

Elevation of MG53 abundance abolishes, whereas decrease of MG53 facilitates, the myogenesis of $\mathrm{C} 2 \mathrm{C} 12$ myoblasts through ubiquitination and degradation of IRS-1 and consequent inhibition of IGF-1 signaling (Lee et al., 2010; Yi et al., 2013). Structurally, the middle and C-terminal regions of IRS-1 interact with the coiled-coil domain of MG53 (Lee et al., 2010). MG53-mediated myogenesis is also evidenced by the enlarged cross-sectional area and higher fatigue resistance in the skeletal muscle of $m g 53^{-1-}$ mice compared with wild-type littermates (Yi et al., 2013).

Nevertheless, Cai and coworkers have shown that MG53 deficiency leads to a progressive age-dependent muscular dystrophy in $m g 53-/-$ mice (Cai et al., 2009a); however, other studies have failed to detect any defects in the soleus, gastrocnemius, and quadriceps of $m g 53^{-1-}$ mice compared with $w t$ littermates (Song et al., 2013; Yi et al., 2013). In general, the role of MG53 in skeletal muscle cannot be simply defined as "good" or "bad"; rather, it is muscle type- or disease condition-dependent. It merits future investigation to determine whether the lack of MG53-induced myopathy is modeland condition-dependent.

In addition to IR and IRS-1, focal adhesion kinase (FAK) is the third identified substrate of MG53 E3 ligase activity. MG53 causes the ubiquitination and degradation of FAK, subsequently contributing to MG53-mediated inhibition of myogenesis (Nguyen et al., 2014).

In overview, MG53 is a novel regulator of myogenesis, and targeting MG53 is a promising avenue for regenerative medicine in skeletal muscle diseases.

MG53 Induces Skeletal Muscle Stiffness. Another mechanism underlying MG53-mediated impairment of skeletal muscle function is through its binding with sarcoplasmic reticulum $\mathrm{Ca}^{2+}$-ATPase 1a. This binding attenuates sarcoplasmic reticulum $\mathrm{Ca}^{2+}$-ATPase 1a activity and subsequently causes muscle stiffness and delayed relaxation (Lee et al., 2012). MG53 has also been found to regulate extracellular $\mathrm{Ca}^{2+}$ entry via Orai1 and intracellular $\mathrm{Ca}^{2+}$ release through Ry receptor 1 during skeletal muscle contraction (Ahn et al., 2016); however, the role of MG53 in muscle electrophysiology merits further study.

\section{MG53 in the Heart}

MG53 Role in Cardiac I/R Injury Protection. The function of MG53 in cardiomyocytes was first reported by Cao and colleagues (2010), who identified MG53 as an important cardioprotective factor. Since adult mammalian cardiomyocytes are terminally differentiated and have a limited ability to regenerate, cardiomyocyte death leads to permanent loss of cardiac functional units and can cause multiple severe cardiac diseases, including myocardial infarction, I/R injury, arrhythmia, cardiac rupture, and heart failure. The most common cause of cardiomyocyte death is blockage of blood supply to the heart (myocardial infarction). Timely restoration of blood flow (i.e., reperfusion) is the best 
intervention to prevent cardiac cell death and injury induced by myocardial infarction; however, reperfusion causes further damage to the myocardium, termed I/R injury. To date, cardiac I/R injury remains a "neglected therapeutic target," and only limited effective therapies for myocardial I/R damage are available (Hausenloy and Yellon, 2013).

Multiple lines of evidence have demonstrated that MG53 protects the heart against I/R injury (Wang et al., 2010; Cao et al., 2010; Zhang et al., 2011; Liu et al., 2015b). Cardiac I/R insult decreases myocardial MG53 protein levels (Cao et al., 2010; Zhang et al., 2011). MG53 deficiency exaggerates I/Rinduced myocardial damage, whereas elevation of MG53 protects cardiomyocytes from oxidative injury (Cao et al., 2010). Interestingly, S-nitrosylation of MG53 at cysteine 144 abolishes I/R-induced downregulation of MG53 by inhibiting oxidation of cysteine 144 (Kohr et al., 2014). Furthermore, systemic delivery of recombinant MG53 alleviates cardiac I/R injury, suggesting that, in addition to intracellular, extracellular MG53 exerts a cardioprotective effect (Liu et al., 2015b).

MG53 plays an indispensable role in ischemic preconditioning (IPC) and postconditioning protection, both of which are the most powerful intrinsic protective machineries for cardiac I/R injury (Murry et al., 1986; Zhao et al., 2003). A lack of MG53 diminishes the cardioprotection conferred by both IPC and postconditioning (Cao et al., 2010; Zhang et al., 2011). It has recently been shown that MG53 may also participate in remote ischemic preconditioning, which is another form of intrinsic cardiac protection (Ma et al., 2016). MG53 abundance in the heart is also upregulated by anesthetic preconditioning with sevoflurane (Ma et al., 2013), indicating that, in addition to ischemic preconditioning, MG53 may play a role in other forms of cardiac preconditioning. Thus, maintenance of cellular MG53 levels is essential for myocardial integrity.

Several mechanisms have been shown to underlie MG53elicited cardiac protection. Molecular signaling mediating IPC and PosC protection is classified into two major pathways: reperfusion injury salvage kinase (RISK, composed of PI3KAkt-GSK3 $\beta$ and ERK1/2 signaling) and survivor activation factor enhancement (SAFE, composed of the activation of tumor necrosis factor- $\alpha$ and the JAK-STAT3 axis) (Crisostomo et al., 2006; Heusch, 2009; Lacerda et al., 2009; Lecour, 2009). MG53 is essential for the activation of RISK pathway, but not the SAFE pathway (Cao et al., 2010; Zhang et al., 2011). MG53 acts as a molecular bridge, binding to the p85 subunit of PI3K with its $\mathrm{N}$ terminus and to $\mathrm{CaV} 3$ with its $\mathrm{C}$ terminus. This dual binding facilitates the formation of the p85-PI3K/MG53/ $\mathrm{CaV} 3$ survival complex, which is indispensable for activation of the RISK pathway (Cao et al., 2010; Zhang et al., 2011). In this way, in addition to its membrane repair function, MG53 serves as a docking/adapter protein for cell signaling to mediate activation of the cell survival signaling pathway.

Another mechanism of cardioprotection by MG53 involves the facilitation of cardiac plasma membrane repair, which is similar to that seen in skeletal muscle (Wang et al., 2010); however, the mechanisms underlying MG53-mediated membrane resealing in cardiomyocyte are different from those in skeletal muscle. Although MG53 does not bind to cholesterolbinding protein in lipid profiling analysis for skeletal muscle, (Cai et al., 2009a), cholesterol is indispensable for the membrane repair function of MG53 in cardiomyocytes (Wang et al., 2010). It is possible that a cholesterol- dependent secondary membrane structure, rather than the physical interaction of cholesterol with MG53, contributes to the membrane repair process. Additionally, in cardiomyocytes, the redox condition is not required for the translocation of MG53, but it is necessary for the stabilization of the nascent MG53 repair patch (Wang et al., 2010). Since the MG53mediated membrane repair process and activation of cell survival signaling occur in or adjacent to lipid rafts or caveolae (Cai et al., 2009a; Wang et al., 2010; Cao et al., 2010; Zhang et al., 2011), it is possible that both mechanisms are involved in MG53-mediated cardioprotection.

Upregulation of MG53 Leads to Diabetic Cardiomyopathy. Similar to the situation in skeletal muscle, MG53 exerts both beneficial and adverse effects in the heart. Diabetic cardiomyopathy is a major complication of diabetes and is the leading cause of morbidity and mortality for diabetic patients (Kannel et al., 1974; Kannel and McGee, 1979a,b,c; Devereux et al., 2000). Recently, it has been demonstrated that MG53 plays a critical role in the development of diabetic cardiomyopathy. In the hearts of multiple animal models with MetS, MG53 protein levels are profoundly upregulated. When specifically overexpressed in the heart, MG53 causes cardiac fibrosis and dysfunction, insulin resistance, depressed glucose uptake, and elevated lipid accumulation, all of which are typical phenotypes of diabetic cardiomyopathy (Liu et al., 2015b). Mechanistically, MG53 not only induces myocardial IR and IRS-1 degradation, insulin resistance, and disorders in glucose metabolism, but it also impaired cardiomyocyte lipid metabolism via transcriptionally upregulated peroxisome proliferation-activated receptor- $\alpha$ (PPAR- $\alpha$ ) and its target genes (Liu et al., 2015b). Increased intracellular MG53 levels lead to excessive cardiac lipid accumulation, which contributes to the pathogenesis of diabetic cardiomyopathy (Liu et al., 2015b). Altogether, MG53 is a key regulator of both glucose and lipid metabolism in the heart.

Additionally, in a recent study, MG53 was expressed in cardiac fibroblasts. Inhibition of MG53 has been shown to prevent proliferation and migration of fibroblasts by regulating transforming growth factor- $\beta$ signaling (Zhao and Lei, 2016). These findings indicate that MG53 may also be involved in cardiac fibrosis and remodeling.

\section{MG53 in Other Tissues}

Although originally thought to be a cardiac and skeletal muscle-specific protein, MG53 is present in nonmuscular organs, including the lungs (Jia et al., 2014; Kim et al., 2014) and kidney (Duann et al., 2015). Regardless of its relatively low expression level $(\sim 2.5 \%-5 \%$ of muscle) (Jia et al., 2014; Kim et al., 2014 Duann et al., 2015), MG53 still plays important physiologic roles in nonmuscle organs.

In the lung, MG53 expression is restricted to type 1 and 2 alveolar epithelial cells; it is absent from smooth muscle and endothelial cells (Jia et al., 2014). MG53 ablation profoundly exaggerates lung injury caused by various stresses, including overventilation and I/R (Jia et al., 2014; Kim et al., 2014). Both transgenic overexpression of endogenous MG53 and exogenous application of recombinant MG53 promote the repair of alveolar epithelial cells and prevent lung injury (Jia et al., 2014; Kim et al., 2014), indicating that MG53 is a promising therapeutic target for acute lung injury. 
In the kidney, MG53 is predominantly expressed in the inner cortex (Duann et al., 2015). In $m g 53^{-1-}$ mice, I/Rinduced kidney damage is markedly exaggerated compared with that in $w t$ animals. More importantly, intravenous injection of recombinant MG53 abolishes the kidney injury induced by both $\mathrm{I} / \mathrm{R}$ and a chemotherapeutic agent, suggesting that MG53 exhibits a renal protective effect (Duann et al., 2015).

In some cultured nonmuscle cells, including keratinocytes, neuronal, and immune origin cells, although there is no endogenous MG53, forced expression of MG53 confers a membrane repair function similar to that seen in striated muscle cells (Weisleder et al., 2012; Yao et al., 2016). In in vivo models, systemic delivery of the recombinant MG53 protein markedly facilitates wound healing and prevents tissue injury (Li et al., 2015; Yao et al., 2016). Exogenous MG53 exerts a protective effect via promotion of membrane repai, as well as activation of the cell survival signaling pathway (Weisleder et al., 2012; Liu et al., 2015b; Yao et al., 2016). These data further support the clinical value of MG53 in human diseases. Both intracellular and extracellular MG53 exert protective effects in striated muscle and nonmuscle tissues.

\section{Regulation of MG53 Transcription: Differences in Humans and Rodents}

The intracellular MG53 level is dynamically regulated in multiple physiologic and pathologic conditions. In the heart, MG53 abundance increases progressively during the embryonic period and until 4 weeks after birth (Wang et al., 2010). The level of MG53 is decreased by I/R injury; this response is largely prevented by IPC protection (Cao et al., 2010). In skeletal muscle, several studies have demonstrated that the protein level of MG53 is elevated during myogenesis (Jung and Ko, 2010; Lee et al., 2010; Yi et al., 2013; Nguyen et al., 2014). In both the heart and skeletal muscle of multiple animal models with insulin resistance and metabolic disorders, MG53 expression is markedly upregulated (Song et al., 2013; Liu et al., 2015a). More importantly, MG53 level is also increased in the skeletal muscle of obese humans (Song et al., 2013). Elevated MG53 and subsequent insulin resistance have been detected in mouse skeletal muscle as early as 1 week after HFD treatment, when insulin sensitivity in the liver and fat was normal (Song et al., 2013). These results suggest that insulin resistance in skeletal muscle, but not in liver or fat, is the initial and primary cause of HFD-induced systemic insulin resistance and that uregulation of MG53 is an important mechanism of skeletal muscle insulin resistance. Upregulation of both MG53 mRNA and protein has been reported in skeletal muscle in HFD-treated animals (Qi et al., 2016), although one study found no change in MG53 protein levels in multiple muscles from mice and humans with metabolic diseases (Yi et al., 2013). This discrepancy may be due to differences in the MG53 antibodies used, different disease models, or complications of drug treatment.

In regard to the regulation of MG53 transcription, it has been reported that MG53 expression is induced during myogenesis by MyoD, which is activated by the PI3K/Akt signaling pathway but is independent of mTOR, FOXO1, or GSK3 $\beta$ (Lee et al., 2010). Myocyte enhancer factor-2 (MEF2) is also able to activate transcription of MG53 (Jung and Ko, 2010). MG53 expression is synergistically upregulated by MEF2 and MyoD (Jung and Ko, 2010). Under pathologic conditions such as insulin resistance and MetS, MEF2, and MyoD are unlikely to be the regulators of MG53 in skeletal muscle since they are either downregulated or unaltered under those conditions (Zhou et al., 2012; Brown et al., 2015; Sato et al., 2015). Other factors may be involved in the regulation of MG53 expression under these circumstances.

To date, there are no published data concerning the transcriptional regulation of MG53 in cardiomyocytes. It should be noted that, according to a recent report, MG53 abundance is quite low in human, ovine, and porcine hearts, although it is abundantly present in rat and mouse hearts (Lemckert et al., 2016). These data suggest that there are species differences in the regulation of cardiac MG53 expression. Future studies are required to delineate the mechanisms responsible for the regulation of MG53 expression, especially under pathologic conditions.

To translate the findings in the basic research of MG53 to clinical diagnosis and therapeutics, it is important to assess differences in MG53 expression in the hearts of humans versus rodent models (Lemckert et al., 2016). The absence of MG53 in human hearts makes it less clinically relevant to suppress cardiac MG53 to prevent cardiomyocyte metabolic disorders and to use plasma MG53 as a biomarker of cardiac injury. At the same time, since there is no endogenous MG53, the human heart presumably will be be more sensitive to cardiac protection in response to exogenous recombinant MG53. In addition, because of the lack of endogenous myocardial MG53 in humans, inhibition of MG53 E3 activity may be a more beneficial potential therapy for type 2 diabetes and its various complications without causing cardiac side effects.

\section{Concluding Remarks}

Since the first report on MG53 biologic function in 2009 (Cai et al., 2009a), multiple physiologic and pathologic roles have been identified for MG53 in both cardiac and skeletal muscle and in nonmuscle organs, indicating potential significance in quite a few important human diseases. MG53 exerts both beneficial and adverse effects on human diseases. Acute elevation of intracellular MG53 protects heart and skeletal muscle against injury via both improved membrane repair (Cai et al., 2009a; Wang et al., 2010) and activation of the protective signaling pathway (Cao et al., 2010; Zhang et al., 2011); however, a sustained increase in MG53 abundance induces insulin resistance (through E3 ligase-dependent degradation) and lipid accumulation (through transcriptional regulation of peroxisome proliferation-activated receptor- $\alpha$ ) (Song et al., 2013; Yi et al., 2013; Liu et al., 2015a). In acute settings, including acute myocardial infarction and cardiac and skeletal muscle I/R injury, a transient and short-term increase of MG53 protects against acute damage and prevents sudden death. To avoid insulin resistance or lipid dysregulation, engineering of the MG53 protein or specifically inactivating its E3 activity may provide a novel strategy for the treatment of metabolic diseases. Abolishment of E3 ligase activity of MG53 prevents its inhibition of myogenesis and skeletal muscle regeneration.

Currently, almost all studies on the functions of MG53 are performed in animal models. Further work should be done to develop this TRIM family member into a novel therapeutic 
for various human diseases, including cardiovascular diseases, skeletal muscle injury, and diabetes and related complications.

\section{Authorship Contributions}

Wrote or contributed to the writing of the manuscript: Zhang, Wu, $\mathrm{Lv}$, Xiao.

\section{References}

Ahn MK, Lee KJ, Cai C, Huang M, Cho CH, Ma J, and Lee EH (2016) Mitsugumin 53 regulates extracellular $\mathrm{Ca}\left({ }^{2+}\right)$ entry and intracellular $\left.\mathrm{Ca}^{(2+}\right)$ release via Orai1 and RyR1 in skeletal muscle. Sci Rep 6:36909.

Baker J, Liu JP, Robertson EJ, and Efstratiadis A (1993) Role of insulin-like growth factors in embryonic and postnatal growth. Cell 75:73-82.

Bansal D and Campbell KP (2004) Dysferlin and the plasma membrane repair in muscular dystrophy. Trends Cell Biol 14:206-213.

Bansal D, Miyake K, Vogel SS, Groh S, Chen CC, Williamson R, McNeil PL, and Campbell KP (2003) Defective membrane repair in dysferlin-deficient muscular dystrophy. Nature 423:168-172.

Brown LA, Lee DE, Patton JF, Perry, JrRA, Brown JL, Baum JI, Smith-Blair N, Greene NP, and Washington TA (2015) Diet-induced obesity alters anabolic signalling in mice at the onset of skeletal muscle regeneration. Acta Physiol (Oxf) 215: $46-57$.

Cai C, Lin P, Zhu H, Ko JK, Hwang M, Tan T, Pan Z, Korichneva I, and Ma J (2015) Zinc binding to MG53 protein facilitates repair of injury to cell membranes. $J$ Biol Chem 290:13830-13839.

Cai C, Masumiya H, Weisleder N, Matsuda N, Nishi M, Hwang M, Ko JK, Lin P, Thornton A, Zhao X, et al. (2009a) MG53 nucleates assembly of cell membrane repair machinery. Nat Cell Biol 11:56-64.

Cai C, Masumiya H, Weisleder N, Pan Z, Nishi M, Komazaki S, Takeshima H, and Ma J (2009b) MG53 regulates membrane budding and exocytosis in muscle cells. J Biol Chem 284:3314-3322.

Cao CM, Zhang Y, Weisleder N, Ferrante C, Wang X, Lv F, Zhang Y, Song R, Hwang M, Jin L, et al. (2010) MG53 constitutes a primary determinant of cardiac ischemic preconditioning. Circulation 121:2565-2574.

Chargé SB and Rudnicki MA (2004) Cellular and molecular regulation of muscle regeneration. Physiol Rev 84:209-238.

Corona BT, Garg K, Roe JL, Zhu H, Park KH, Ma J, and Walters TJ (2014) Effect of recombinant human MG53 protein on tourniquet-induced ischemia-reperfusion injury in rat muscle. Muscle Nerve 49:919-921.

Crisostomo PR, Wairiuko GM, Wang M, Tsai BM, Morrell ED, and Meldrum DR (2006) Preconditioning versus postconditioning: mechanisms and therapeutic potentials. J Am Coll Surg 202:797-812.

DeFronzo RA, Jacot E, Jequier E, Maeder E, Wahren J, and Felber JP (1981) The effect of insulin on the disposal of intravenous glucose. Results from indirect calorimetry and hepatic and femoral venous catheterization. Diabetes 30:1000-1007.

Devereux RB, Roman MJ, Paranicas M, O'Grady MJ, Lee ET, Welty TK, Fabsitz RR, Robbins D, Rhoades ER, and Howard BV (2000) Impact of diabetes on cardiac structure and function: the strong heart study. Circulation 101:2271-2276.

Doyle JM, Gao J, Wang J, Yang M, and Potts PR (2010) MAGE-RING protein complexes comprise a family of E3 ubiquitin ligases. Mol Cell 39:963-974

Duann P, Li H, Lin P, Tan T, Wang Z, Chen K, Zhou X, Gumpper K, Zhu H, Ludwig T, et al. (2015) MG53-mediated cell membrane repair protects against acute kidney injury. Sci Transl Med 7:279ra36 DOI: 10.1126/scitranslmed.

Eckel RH, Grundy SM, and Zimmet PZ (2005) The metabolic syndrome. Lancet 365 : $1415-1428$.

Glass DJ (2003) Molecular mechanisms modulating muscle mass. Trends Mol Med 9: 344-350.

Goodyear LJ, Giorgino F, Sherman LA, Carey J, Smith RJ, and Dohm GL (1995) Insulin receptor phosphorylation, insulin receptor substrate-1 phosphorylation, and phosphatidylinositol 3-kinase activity are decreased in intact skeletal muscle strips from obese subjects. J Clin Invest 95:2195-2204.

Grundy SM (2006) Metabolic syndrome: connecting and reconciling cardiovascular and diabetes worlds. $J$ Am Coll Cardiol 47:1093-1100.

Han R and Campbell KP (2007) Dysferlin and muscle membrane repair. Curr Opin Cell Biol 19:409-416.

Hatakeyama S (2011) TRIM proteins and cancer. Nat Rev Cancer 11:792-804.

Hausenloy DJ and Yellon DM (2013) Myocardial ischemia-reperfusion injury: a neglected therapeutic target. J Clin Invest 123:92-100.

He B, Tang RH, Weisleder N, Xiao B, Yuan Z, Cai C, Zhu H, Lin P, Qiao C, Li J, et al. (2012) Enhancing muscle membrane repair by gene delivery of MG53 ameliorates muscular dystrophy and heart failure in $\delta$-Sarcoglycan-deficient hamsters. Mol Ther 20:727-735.

Hershko A and Ciechanover A (1998) The ubiquitin system. Annu Rev Biochem 67 $425-479$.

Heusch G (2009) No risk, no ... cardioprotection? A critical perspective. Cardiovasc Res 84:173-175.

Huibregtse JM, Scheffner M, Beaudenon S, and Howley PM (1995) A family of proteins structurally and functionally related to the E6-AP ubiquitin-protein ligase. Proc Natl Acad Sci USA 92:2563-2567.

Hwang M, Ko JK, Weisleder N, Takeshima H, and Ma J (2011) Redox-dependent oligomerization through a leucine zipper motif is essential for MG53-mediated cell membrane repair. Am J Physiol Cell Physiol 301:C106-C114.

Jia Y, Chen K, Lin P, Lieber G, Nishi M, Yan R, Wang Z, Yao Y, Li Y, Whitson BA et al. (2014) Treatment of acute lung injury by targeting MG53-mediated cell membrane repair. Nat Commun 5:4387.
Joazeiro CA and Weissman AM (2000) RING finger proteins: mediators of ubiquitin ligase activity. Cell 102:549-552.

Jung SY and Ko YG (2010) TRIM72, a novel negative feedback regulator of myogenesis, is transcriptionally activated by the synergism of MyoD (or myogenin) and MEF2. Biochem Biophys Res Commun 396:238-245.

Kannel WB, Hjortland M, and Castelli WP (1974) Role of diabetes in congestive heart failure: the Framingham study. Am J Cardiol 34:29-34.

Kannel WB and McGee DL (1979a) Diabetes and cardiovascular disease: the Framingham study. JAMA 241:2035-2038.

Kannel WB and McGee DL (1979b) Diabetes and cardiovascular risk factors: the Framingham study. Circulation 59:8-13.

Kannel WB and McGee DL (1979c) Diabetes and glucose tolerance as risk factors for cardiovascular disease: the Framingham study. Diabetes Care 2:120-126.

Kerouz NJ, Hörsch D, Pons S, and Kahn CR (1997) Differential regulation of insulin receptor substrates-1 and -2 (IRS-1 and IRS-2) and phosphatidylinositol 3-kinase isoforms in liver and muscle of the obese diabetic (ob/ob) mouse. J Clin Invest 100: 3164-3172.

Kido Y, Burks DJ, Withers D, Bruning JC, Kahn CR, White MF, and Accili D (2000) Tissue-specific insulin resistance in mice with mutations in the insulin receptor, IRS-1, and IRS-2. J Clin Invest 105:199-205.

Kim SC, Kellett T, Wang S, Nishi M, Nagre N, Zhou B, Flodby P, Shilo K, Ghadiali SN, Takeshima H, et al. (2014) TRIM72 is required for effective repair of alveolar epithelial cell wounding. Am J Physiol Lung Cell Mol Physiol 307:L449-L459.

Kohr MJ, Evangelista AM, Ferlito M, Steenbergen C, and Murphy E (2014) Snitrosylation of TRIM72 at cysteine 144 is critical for protection against oxidationinduced protein degradation and cell death. J Mol Cell Cardiol 69:67-74.

Lacerda L, Somers S, Opie LH, and Lecour S (2009) Ischaemic postconditioning protects against reperfusion injury via the SAFE pathway. Cardiovasc Res 84: 201-208.

Lecour S (2009) Activation of the protective survivor activating factor enhancement (SAFE) pathway against reperfusion injury: does it go beyond the RISK pathway? J Mol Cell Cardiol 47:32-40.

Lee CS, Yi JS, Jung SY, Kim BW, Lee NR, Choo HJ, Jang SY, Han J, Chi SG, Park M, et al. (2010) TRIM72 negatively regulates myogenesis via targeting insulin receptor substrate-1. Cell Death Differ 17:1254-1265.

Lee H, Park JJ, Nguyen N, Park JS, Hong J, Kim SH, Song WY, Kim HJ, Choi K, Cho $\mathrm{S}$, et al. (2016) MG53-IRS-1 interaction disruptor sensitizes insulin signaling in skeletal muscle. J Biol Chem DOI: 10.1074/jbc.M116.754424.

Lee KJ, Park CS, Woo JS, Kim DH, Ma J, and Lee EH (2012) Mitsugumin 53 attenuates the activity of sarcoplasmic reticulum $\mathrm{Ca}\left({ }^{2+}\right)$-ATPase 1a (SERCA1a) in skeletal muscle. Biochem Biophys Res Commun 428:383-388.

Lemckert FA, Bournazos A, Eckert DM, Kenzler M, Hawkes JM, Butler TL, Ceely B North KN, Winlaw DS, Egan JR, et al. (2016) Lack of MG53 in human heart precludes utility as a biomarker of myocardial injury or endogenous cardioprotective factor. Cardiovasc Res 110:178-187.

Li H, Duann P, Lin PH, Zhao L, Fan Z, Tan T, Zhou X, Sun M, Fu M, Orange M, et al. (2015) Modulation of wound healing and scar formation by MG53 protein-mediated cell membrane repair. J Biol Chem 290:24592-24603.

Li Y, Wu H, Wu W, Zhuo W, Liu W, Zhang Y, Cheng M, Chen YG, Gao N, Yu H, et al. (2014) Structural insights into the TRIM family of ubiquitin E3 ligases. Cell Res 24: $762-765$.

Liu F, Song R, Feng Y, Guo J, Chen Y, Zhang Y, Chen T, Wang Y, Huang Y, Li CY, et al. (2015a) Upregulation of MG53 induces diabetic cardiomyopathy through transcriptional activation of peroxisome proliferation-activated receptor $\alpha$. Circulation 131:795-804.

Liu J, Zhu H, Zheng Y, Xu Z, Li L, Tan T, Park KH, Hou J, Zhang C, Li D, et al. (2015b) Cardioprotection of recombinant human MG53 protein in a porcine model of ischemia and reperfusion injury. J Mol Cell Cardiol 80:10-19.

Long YC, Cheng Z, Copps KD, and White MF (2011) Insulin receptor substrates Irs1 and Irs2 coordinate skeletal muscle growth and metabolism via the Akt and AMPK pathways. Mol Cell Biol 31:430-441.

Lorick KL, Jensen JP, Fang S, Ong AM, Hatakeyama S, and Weissman AM (1999) RING fingers mediate ubiquitin-conjugating enzyme (E2)-dependent ubiquitination. Proc Natl Acad Sci USA 96:11364-11369.

Ma LL, Kong FJ, Guo JJ, Zhu JB, Shi HT, Li Y, Sun RH, and Ge JB (2016) Hypercholesterolemia abrogates remote ischemic preconditioning-induced cardioprotection: role of reperfusion injury salvage kinase signals. Shock 47:363269.

Ma LL, Zhang FJ, Qian LB, Kong FJ, Sun JF, Zhou C, Peng YN, Xu HJ, Wang WN, Wen CY, et al. (2013) Hypercholesterolemia blocked sevoflurane-induced cardioprotection against ischemia-reperfusion injury by alteration of the MG53/RISK/ GSK3 $\beta$ signaling. Int $J$ Cardiol 168:3671-3678.

McMillen IC and Robinson JS (2005) Developmental origins of the metabolic syndrome: prediction, plasticity, and programming. Physiol Rev 85:571-633.

Meroni G and Diez-Roux G (2005) TRIM/RBCC, a novel class of 'single protein RING finger' E3 ubiquitin ligases. BioEssays 27:1147-1157.

Munir M (2010) TRIM proteins: another class of viral victims. Sci Signal 3:jc2.

Murry CE, Jennings RB, and Reimer KA (1986) Preconditioning with ischemia: a delay of lethal cell injury in ischemic myocardium. Circulation 74:1124-1136.

Nguyen N, Yi JS, Park H, Lee JS, and Ko YG (2014) Mitsugumin 53 (MG53) ligase ubiquitinates focal adhesion kinase during skeletal myogenesis. J Biol Chem 289: $3209-3216$

Olefsky J, Bacon VC, and Baur S (1976) Insulin receptors of skeletal muscle: specific insulin binding sites and demonstration of decreased numbers of sites in obese rats. Metabolism 25:179-191.

Ozato K, Shin DM, Chang TH, and Morse, 3rdHC (2008) TRIM family proteins and their emerging roles in innate immunity. Nat Rev Immunol 8:849-860.

Powell-Braxton L, Hollingshead P, Warburton C, Dowd M, Pitts-Meek S, Dalton D, Gillett N, and Stewart TA (1993) IGF-I is required for normal embryonic growth in mice. Genes Dev 7:2609-2617. 
Qi J, Yang B, Ren C, Fu J, and Zhang J (2016) Swimming exercise alleviated insulin resistance by regulating tripartite motif family protein 72 expression and AKT signal pathway in Sprague-Dawley rats fed with high-fat diet. J Diabetes Res 2016: 1564386 .

Rudnicki MA, Le Grand F, McKinnell I, and Kuang S (2008) The molecular regulation of muscle stem cell function. Cold Spring Harb Symp Quant Biol 73:323-331.

Sato S, Ogura Y, Tajrishi MM, and Kumar A (2015) Elevated levels of TWEAK in skeletal muscle promote visceral obesity, insulin resistance, and metabolic dysfunction. FASEB J 29:988-1002.

Scheffner M, Nuber U, and Huibregtse JM (1995) Protein ubiquitination involving an E1-E2-E3 enzyme ubiquitin thioester cascade. Nature 373:81-83.

Shulman GI, Rothman DL, Jue T, Stein P, DeFronzo RA, and Shulman RG (1990) Quantitation of muscle glycogen synthesis in normal subjects and subjects with non-insulin-dependent diabetes by $13 \mathrm{C}$ nuclear magnetic resonance spectroscopy. N Engl J Med 322:223-228.

Song R, Peng W, Zhang Y, Lv F, Wu HK, Guo J, Cao Y, Pi Y, Zhang X, Jin L, et al. (2013) Central role of E3 ubiquitin ligase MG53 in insulin resistance and metabolic disorders. Nature 494:375-379.

Wang C, Wang H, Wu D, Hu J, Wu W, Zhang Y, and Peng X (2016) A novel perspective for burn-induced myopathy: Membrane repair defect. Sci Rep 6:31409.

Wang X, Xie W, Zhang Y, Lin P, Han L, Han P, Wang Y, Chen Z, Ji G, Zheng M, et al (2010) Cardioprotection of ischemia/reperfusion injury by cholesterol-dependent MG53-mediated membrane repair. Circ Res 107:76-83.

Weisleder N, Takeshima H, and Ma J (2008) Immuno-proteomic approach to excitation-contraction coupling in skeletal and cardiac muscle: molecular insights revealed by the mitsugumins. Cell Calcium 43:1-8.

Weisleder N, Takizawa N, Lin P, Wang X, Cao C, Zhang Y, Tan T, Ferrante C, Zhu H, Chen PJ, et al. (2012) Recombinant MG53 protein modulates therapeutic cel membrane repair in treatment of muscular dystrophy. Sci Transl Med 4: $139 \mathrm{ra} 85$.

Yao Y, Zhang B, Zhu H, Li H, Han Y, Chen K, Wang Z, Zeng J, Liu Y, Wang X, et al. (2016) MG53 permeates through blood-brain barrier to protect ischemic brain injury. Oncotarget 7:22474-22485.

Yi JS, Park JS, Ham YM, Nguyen N, Lee NR, Hong J, Kim BW, Lee H, Lee CS, Jeong BC, et al. (2013) MG53-induced IRS-1 ubiquitination negatively regulates skeletal myogenesis and insulin signalling. Nat Commun 4:2354.

Zhang Y, Lv F, Jin L, Peng W, Song R, Ma J, Cao CM, and Xiao RP (2011) MG53 participates in ischaemic postconditioning through the RISK signalling pathway. Cardiovasc Res 91:108-115.

Zhao $\mathrm{J}$ and Lei H (2016) Tripartite motif protein 72 regulates the proliferation and migration of rat cardiac fibroblasts via the transforming growth factor- $\beta$ signaling pathway. Cardiology 134:340-346.

Zhao ZQ, Corvera JS, Halkos ME, Kerendi F, Wang NP, Guyton RA, and VintenJohansen $J$ (2003) Inhibition of myocardial injury by ischemic postconditioning during reperfusion: comparison with ischemic preconditioning. Am J Physiol Heart Circ Physiol 285:H579-H588.

Zhou D, Strakovsky RS, Zhang X and Pan YX (2012) The skeletal muscle Wnt pathway may modulate insulin resistance and muscle development in a dietinduced obese rat model. Obesity 20:1577-1584.

Zhu H, Hou J, Roe JL, Park KH, Tan T, Zheng Y, Li L, Zhang C, Liu J, Liu Z, et al. (2015) Amelioration of ischemia-reperfusion-induced muscle injury by the recombinant human MG53 protein. Muscle Nerve 52:852-858.

Address correspondence to: Dr. Yan Zhang, Institute of Molecular Medicine, Peking University, Beijing 100871, China. E-mail: zhangyan9876@pku.edu.cn 